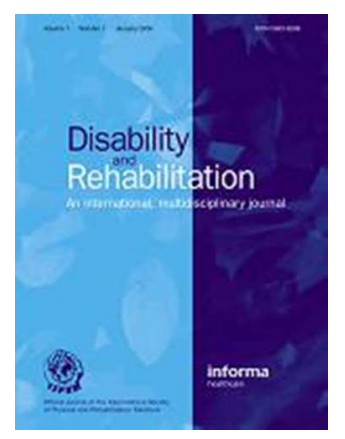

\title{
A systematic review: The influence of real time feedback on wheelchair propulsion biomechanics
}

\begin{tabular}{|r|l|}
\hline Journal: & Disability and Rehabilitation \\
\hline Manuscript ID & Draft \\
\hline Manuscript Type: & Review \\
\hline Keywords: & $\begin{array}{l}\text { Manual wheelchair propulsion, Mechanical effective force, Peak force, Push } \\
\text { arc, Real time feedback, Shoulder injury }\end{array}$ \\
\hline \multicolumn{2}{|c}{} \\
\hline
\end{tabular}




\title{
A systematic review: The influence of real time feedback on wheelchair propulsion biomechanics
}

\author{
Background: Clinical guidelines recommend that, in order to minimize upper \\ limb injury risk, wheelchair users adopt a semi-circular pattern with a slow \\ cadence and a large push arc \\ Objectives: To examine whether real time feedback can be used to influence \\ manual wheelchair propulsion biomechanics. \\ Review methods: Clinical trials and case series comparing the use of real time \\ feedback against no feedback were included. A general review was performed \\ and methodological quality assessed by two independent practitioners using the \\ Downs and Black checklist. The review was completed in accordance with the \\ PRISMA guidelines.
}

Results: Six papers met the inclusion criteria. Selected studies involved 123 participants and analysed the effect of visual and, in one case, haptic feedback. Across the studies it was shown that participants were able to achieve significant changes in propulsion biomechanics, when provided with real time feedback. However, the effect of targeting a single propulsion variable might lead to unwanted alterations in other parameters. Methodological assessment identified weaknesses in external validity.

Conclusions: Visual feedback could be used to consistently increase push arc and decrease push rate, and may be the best focus for feedback training. Further investigation is required to assess such intervention during outdoor propulsion.

Keywords: Manual wheelchair propulsion, mechanical effective force, peak force, push arc, real time feedback, shoulder injury

\section{Introduction}

Sustained manual wheelchair propulsion commonly leads to upper limb injury and pain, which is associated with reduced physical activity and quality of life [1]. Published clinical guidelines suggest that manual wheelchair users should aim to minimise peak force and repetition during completion of a task [2]. To achieve this, in terms of 
propulsion biomechanics, manual wheelchair users are commonly advised to propel with a semicircular pattern [3] at a push rate of 1 push per second and push arc in the range of $85^{\circ}$ to $100^{\circ}[4]$

Wheelchair skills training has demonstrated benefit to manual wheelchair users, leading to an improvement in ability to complete a variety of functional tasks $[5,6]$. Tracking and modification of specific propulsion parameters can be optimised with the use of instrumented wheelchair wheels, which have the capacity to measure the temporal parameters of propulsion in addition to the 3-dimensional forces and moments applied by the user to the wheelchair push rim [7]. The output from such devices has the potential to provide real time feedback during manual wheelchair propulsion. Similar real time biofeedback interventions have been previously proved successful in helping athletes modifying movement strategies, thus reducing injury risk $[8,9]$.

The aim of this systematic review is to examine the current knowledge about the benefit of using real time feedback to modify wheelchair propulsion biomechanics. The review will consider different types of feedback and their impact on both temporal and kinetic propulsion parameters. As instrumented wheelchair wheels and other rehabilitation devices become more widely available, it is important to identify how optimising methods of real time feedback could improve propulsion efficiency and minimise injury risk.

\section{Methods}

\section{Study selection process}

A systematic review was completed to assess the influence of real time feedback on wheelchair propulsion biomechanics. The design of the review was developed according to the guidelines provided in the Preferred Reporting Items for Systematic 
Reviews and Meta Analyses (PRISMA) statement [10]. The electronic databases Web of Science, PubMed, Science Direct, Cochrane Database of Systematic Reviews and IEEE Xplore were searched, including their full archive history to December 2015, using the following search terms:

Manual wheelchair propulsion AND feedback

The titles and abstracts of all studies identified were screened by two independent reviewers, and if matching the review inclusion and exclusion criteria full text articles were obtained. The reference list of all selected full text articles was also reviewed. The inclusion criteria for the review were as follows:

(1) Clinical trials and case series comparing the effect of real time feedback and no real time feedback on wheelchair propulsion biomechanics

(2) Clinical trials including real time verbal, visual and haptic feedback

(3) Full text, English language publications

(4) Experienced and novice wheelchair users of any age

The exclusion criteria for the review were as follows:

(1) Case studies, editorials and review articles

(2) Studies not comparing real time feedback to no real time feedback

(3) Non-English language articles

(4) Unpublished theses and dissertations

Significant data from all included studies were extracted by both reviewers and subsequently compared in order to ensure completeness and consistency. Extracted data included number and characteristics of participants, study design, type and length of intervention, mean and standard deviation of outcome measures assessed and timing of post intervention assessment. 


\section{Study review process}

A general review of the literature was completed, including assessment of study design, study population, the type of real time feedback provided, the outcome measures used and whether the main findings were statistically significant. In addition, the methodological quality of each of the studies was assessed using a modified version of the checklist published by Downs and Black [11]. The checklist has been previously used to assess the methodological quality of similar studies [12]. The checklist scores methodological quality under the headings reporting, external validity, internal validity bias, internal validity confounding and power. The question relating to study power was simplified to determine whether a power calculation was performed. If the answer was 'yes' one point was awarded and if 'no', zero points were awarded. Each article was reviewed against the checklist by two people working independently. Results were then compared, and disagreements were resolved during a face to face discussion.

\section{Results}

\section{Study selection}

The systematic review identified 281 citations. On review of the title and abstract of these citations, 18 articles were considered appropriate for full review and full text versions obtained. 12 of these articles were excluded. One was a case study, four studies did not assess an intervention, and seven provided an intervention to improve wheelchair propulsion but did not examine the implementation of real time feedback.

Six articles met the inclusion and exclusion criteria for the review. The review process is illustrated in Figure 1 and a summary of the main characteristics of the included studies is provided in Table 1.

Figure 1.

Table 1. 


\section{Participants}

In total, 123 participants were assessed in the six studies, 109 being male and 14 female. The mean age across the six studies calculated from the mean values presented was 35.5 years. 5 studies examined a total of 103 experienced manual wheelchair users [13-17], the other study examined 20 novice non wheelchair users [18]. The 103 experienced manual wheelchair users comprised 92 participants with a diagnosis of Spinal Cord Injury (Injury level range C6-L3), six with a diagnosis of Spina Bifida, two with a diagnosis of Cerebral Palsy and single participants with a diagnosis of Spinal Lipoma, Multiple Sclerosis and Spinal Muscular Atrophy. The mean time as a manual wheelchair user calculated from these 103 experienced participants was 14.6 years.

\section{Study characteristics}

\section{Study design}

Two of the studies were randomised controlled trials $[14,18]$. The remainder of the studies employed a repeated measures design, assessing the change in propulsion biomechanics following intervention with respect to pre-intervention 'control' biomechanical results $[13,15-17]$.

\section{Intervention}

The studies used interventions including haptic, verbal and visual feedback. Only one of the studies examined haptic feedback [13]. This was delivered by a wheelchair simulator, on to which a wheelchair was positioned. Haptic feedback was delivered via an increase in resistance to propulsion when participants deviated from the suggested mechanical effective force (MEF). Participants were also provided with visual feedback to ensure maintenance of propulsion velocity. One of the randomised controlled trials divided participants in to three groups; a control group, an instruction 
only group that received a multimedia presentation and an intervention group that received real time visual feedback on push frequency, push arc and propulsion velocity in addition to the multimedia presentation [14]. The other randomised controlled trial divided the participants into two groups, a control group receiving only real time visual feedback on propulsion velocity and an intervention group receiving real time visual feedback on both propulsion velocity and fraction of effective force (FEF) [18]. The remaining studies investigated real time visual feedback focusing on a range of variables. Richter investigated the influence of single variable visual feedback including braking moment, push rate, push arc, push force, push distance and smoothness [15]. DeGroot provided visual feedback on push rate, push arc and push force [16] and Kotajarvi provided visual feedback on FEF, propulsion velocity and power output [17].

\section{Study setting}

Each of the studies was completed in a laboratory setting. Blouin et al. provided both feedback and measured outcome during propulsion on a simulator [13]. Rice et al. provided visual feedback during propulsion on a dynamometer and measured outcome during over ground propulsion [14]. DeGroot et al. provided visual feedback during propulsion on an ergometer and measured outcome during both ergometer and over ground propulsion [16]. The remaining three studies provided both visual feedback and measured outcome during propulsion on an ergometer $[15,17,18]$. Four of the studies measured outcome during the intervention $[13,15,17,18]$. Three of the studies measured outcome immediately post intervention $[13,14,16]$ and only one of the studies presented results from longer term (three months) follow up [14]. 


\section{Outcome measures}

\section{Temporal parameters}

Push rate is defined as the number of push cycles per second. The aim of the interventions reported was to decrease push rate. Three of the studies provided feedback on push rate and recorded it as an outcome measure [14-16]. Rice et al. reported a decrease in push rate in the intervention versus control group at both short term follow up $\left(0.82 \mathrm{sec}^{-1}\right.$ vs. $\left.1.10 \mathrm{sec}^{-1}, \mathrm{P}<0.05\right)$ and long term follow up $\left(0.87 \mathrm{sec}^{-1} \mathrm{vs}\right.$. $\left.1.10 \mathrm{sec}^{-1}, \mathrm{P}<0.05\right)$ [11]. Although the visual feedback group demonstrated no significant reduction in push rate compared to the instruction only group in the short term, at longer term follow up a significant reduction was demonstrated $\left(0.87 \mathrm{sec}^{-1} \mathrm{vs}\right.$. $\left.0.93 \mathrm{sec}^{-1}, \mathrm{P}<0.05\right)$. Richter et al. demonstrated a significant reduction in push rate when both aiming for a maximum reduction $(64 \%$ decrease, $\mathrm{P}<0.005)$ and also a $10 \%$ reduction ( $9 \%$ decrease, $\mathrm{P}<0.005)$ [12]. DeGroot et al. reported a significant reduction in push rate with the addition of visual feedback $\left(0.68 \mathrm{sec}^{-1}\right.$ vs. $\left.0.99 \mathrm{sec}^{-1}, \mathrm{P}<0.01\right)$ [16]. Kotajarvi et al. used push rate as an outcome measure, but did not provide real time feedback on push rate as an intervention [17].

Push arc is defined as the angle over which force is applied to the wheelchair push rim. The aim of the interventions was to increase push arc. Three of the studies provided feedback on push arc and recorded it as an outcome measure [14-16]. Rice et al. reported an increase in push arc in the intervention versus control group at both short term follow up $\left(107.7^{\circ}\right.$ vs. $\left.97.9^{\circ}, \mathrm{P}<0.05\right)$ and long term follow up $\left(111.8^{\circ}\right.$ vs. $97.9^{\circ}$, $\mathrm{P}<0.05$ ) [14]. Although the visual feedback group demonstrated no significant increase in push arc compared to the instruction only group in the short term, there was a significant increase at longer term follow up $\left(111.8^{\circ}\right.$ vs. $\left.104.6^{\circ}, \mathrm{P}<0.05\right)$. Richter et al. demonstrated a significant increase in push arc when aiming for a maximum increase 
(31\% increase, $\mathrm{P}<0.005)$ and also a $10 \%$ increase (10\% increase, $\mathrm{P}<0.005)[15]$.

DeGroot et al. reported a significant increase in push arc with the addition of visual feedback $\left(86.1^{\circ}\right.$ vs. $\left.67.0^{\circ}, \mathrm{P}<0.05\right)[16]$.

\section{Kinetic parameters}

Peak resultant propulsion force describes the total force applied to the wheelchair push rim. The aim of the intervention is to minimise this force. Two of the studies provided feedback on peak force and recorded peak force as an outcome measure $[15,16]$.

Richter et al. reported that participants were able to significantly reduce peak forces when aiming for maximum reduction $(-11 \%, \mathrm{P}<0.005)$, but not when aiming for a $10 \%$ reduction [15]. DeGroot et al. reported a significant increase in peak push force (13.89 pounds vs. 11.89 pounds, $\mathrm{P}<0.05$ ), despite the aim of the feedback being to reduce peak force [16].

Braking moment is defined as the 'minimum (negative) moment about the axle from the end of the previous push phase to the end of the current push phase' [15]. Richter et al. reported a significant reduction in braking moment as a result of visual feedback $(-44 \%, \mathrm{P}<0.005)[15]$.

$\mathrm{MEF} / \mathrm{FEF}$ are defined as the effective component of the propulsion force which drives the wheels forward [17]. Three of the studies provided feedback on MEF/FEF and record $\mathrm{MEF} / \mathrm{FEF}$ as an outcome measure $[13,17,18]$. Blouin et al. reported a significant increase in MEF with the addition of haptic feedback $(\mathrm{P}<0.02)$ [13].

Kotajarvi et al. reported no significant change in FEF at 2 different intensity levels [17]. Contrary to this, de Groot et al. reported significantly greater levels of FEF with feedback at three different levels of power output, $0.15 \mathrm{~W} k \mathrm{~kg}-1$ (90.22\% vs. $79.26 \%$, $\mathrm{P}<0.01), 0.25{\mathrm{~W} k \mathrm{~kg}^{-1}}^{-197.47 \%}$ vs. $\left.83.04 \%, \mathrm{P}<0.01\right)$ and at $0.40{\mathrm{~W} \cdot \mathrm{kg}^{-1}}^{(96.56 \%}$ vs. $83.14 \%, \mathrm{P}<0.01)[18]$. 
Push distance is defined as the distance travelled during one propulsion cycle [15]. Richter et al. reported a significant increase in push distance with visual feedback when aiming for both maximum increase $(255 \%, \mathrm{P}<0.005)$ and also a $10 \%$ increase $(11 \%, \mathrm{P}<0.005)[15]$.

Smoothness is defined as the mean force divided by the peak force (unit less variable)[15]. Richter et al. reported no significant improvement in smoothness with the addition of visual feedback [15].

Four of the studies also provided visual feedback on propulsion velocity $[13,14,17,18]$. This feedback was provided to enable participants to control their velocity, rather than alter it.

\section{Cross variable effects}

One of the studies directly compared the cross variable effect of modifying single variables with visual feedback [15]. Minimising push rate was associated with an increase in contact angle and push distance, but a $154 \%$ increase in peak force.

Maximising push arc was associated with a significant reduction in push rate and an increase in push distance, but a $34 \%$ increase in peak force.

\section{Methodological quality}

The Downs and Black study quality scores are presented in table 2. The highest score was $19 / 28$ [18] and the lowest 12/28 [13]. Across each of the six studies, the scores were particularly low for the section measuring external validity, with all studies completed in the laboratory setting.

Table 2 . 


\section{Discussion}

This systematic review aimed to determine whether the use of real time feedback could lead to changes in manual wheelchair propulsion biomechanics. The results suggest that real time visual feedback can be used to alter push rate [14-16], push arc [14-16], push force [15], MEF [18], braking moment [15] and push distance [15]. The results also suggest that real time haptic feedback can be used to alter MEF [13]. The results suggested that modifying temporal parameters may be more successful than modifying kinetic parameters. There is limited evidence to support the carryover of such interventions, and further research is required to enable useful application of real time feedback away from the laboratory during day to day wheelchair propulsion.

\section{Outcome measures}

Temporal parameters: Reducing push rate has been associated with a reduction in upper extremity total muscle power during a study utilising forward dynamic simulations [19] and also preservation of median nerve function at the wrist $[20,21]$. Increasing push arc has been advised, to enable greater power generation for a set force by applying this force over a greater angle [22]. Providing real time visual feedback to reduce push rate and increase push arc demonstrated beneficial effects during the intervention [15], immediately following the intervention $[14,16]$ and at three months follow up [14], indicating that they may be successful parameters to target as part of an initial training program and also during real time feedback via an instrumented wheelchair wheel.

Kinetic parameters: Higher push rim forces have been associated with both progressive shoulder joint pathology [23] and reduced median nerve function[20]. Guidelines suggest that peak force applied to the push rim should be minimised to 
preserve upper limb function $[2,4]$. The results of the review demonstrated conflicting evidence regarding the use of visual feedback to minimise peak force. DeGroot et al. reported a significant increase in push force [16]. During this study, visual feedback was provided on three variables at the same time (push rate, push arc and peak force) and it was concluded that push force may have increased to compensate for a reduction in push rate to maintain the same push length. Richter et al. reported a significant reduction in push force when participants were attempting to minimise it, but participants were not able to control a reduction in push force of $10 \%$ [15]. This study investigated single variable feedback and discussed the difficulty in minimising peak force, suggesting that providing visual feedback on the whole force curve rather that peak value may be beneficial. The review also identified contrasting results from the studies reporting MEF/FEF as an outcome measure. Blouin et al. reported a significant increase in MEF with the addition of haptic feedback [13] and de Groot et al. reported significant increases in FEF at three levels of power output with the addition of visual feedback [18]. However, Kotajarvi et al. reported no significant increase in FEF at two levels of power output with the addition of visual feedback [17]. In addition to these inconsistencies, the validity of aiming for an increase in MEF/FEF to minimise upper limb injury risk has been questioned. Previous research has highlighted that increased application of tangential force can lead to increased forces and moments at the glenohumeral joint [24] and also increased glenohumeral joint muscle demand [25]. In addition to the greater stresses placed on the upper limb, increasing MEF has been associated with a greater physiological cost [18].

\section{Cross variable effects}

The success of optimising a single variable cannot be measured in isolation of the cross effect on other variables. Only one of the studies reviewed measured 
statistically the impact of altering a single variable on others measured [15]. The results of this study demonstrated that while inducing a desired change such as reducing push rate, there may be a resultant undesirable change, in this case an increase in push force.

To highlight the balance between minimising task repetition and peak force application, it is useful to apply the examples of reducing push rate and increasing push arc to the average daily activity of a manual wheelchair user. Previous data tracking activity levels of manual wheelchair users has reported the average distance travelled per day to be $1600 \mathrm{~m}$ [26]. Using the baseline data and percentage change values for single variable feedback presented by Richter et al. the balance between frequency and load would vary as follows:

Minimising push rate to 18.87 stokes per minute increased push arc to $108.79^{\circ}$, increasing peak force to $145.75 \mathrm{~N}$ with an average distance per push increasing to $4.27 \mathrm{~m}$, the manual wheelchair user would make 374 pushes during the day. Reducing push rate by $10 \%$ to 47.69 strokes per minute increased push arc to $87.90^{\circ}$, increasing peak force to $61.97 \mathrm{~N}$ with an average distance per push increasing to $1.48 \mathrm{~m}$, the manual wheelchair user would make 1082 pushes per day.

Maximising push arc to $114.00^{\circ}$ reduced push rate to 36.69 strokes per minute, increasing peak force to $76.89 \mathrm{~N}$ with an average distance per push of $2.19 \mathrm{~m}$, the manual wheelchair user would make 729 pushes per day. Increasing push arc by $10 \%$ to $95.73^{\circ}$ reduced push rate to 45.07 strokes per minute, increasing peak force to $63.12 \mathrm{~N}$ with an average distance per push of $1.61 \mathrm{~m}$, the manual wheelchair user would make 994 pushes per day.

Minimising the push rate leads to the requirement of many fewer pushes, but the peak forces are very high, equivalent to climbing a $12 \%$ ramp, which are associated with higher glenohumeral joint contact forces and theoretically greater risk of injury 
[27]. Maximising push arc leads to the requirement of fewer pushes, with less increase in peak force, but increasing the push arc to such an extent may lead to injury due to the upper limb moving to greater extremes of movement, which should be avoided [2]. Inducing a $10 \%$ reduction in push rate lead to an increase in peak force and push distance, whereas inducing a $10 \%$ increase in push arc lead to a slighter greater increase in push force than during the push rate reduction, but also a greater increase in push distance and therefore reduced pushes during daily activity. These results suggest that optimising push arc towards $100^{\circ}$ may result in the best balance between peak force and task repetition, although such an assumption needs to be tested during more challenging propulsion tasks away from the laboratory, whilst maintaining the required chair velocity.

\section{Methodological review}

The results revealed that a key future development would be to improve external validity. Each of the studies was completed within a laboratory, with the real time feedback provided during propulsion on an ergometer or treadmill. Propelling a wheelchair outdoors provides a different challenge, negotiating terrain including cross slopes [28] and inclines [29,30] has been shown to increase upper limb demand. Further research is required not only to assess whether real time feedback can be successful in a changing environment, but also to determine how best to apply this feedback. Providing real time visual feedback is possible in a laboratory experiment, but not practical during outdoor propulsion when negotiating the environment requires visual focus on the terrain. The acceptability and effectiveness of other forms of feedback such as auditory and haptic (vibration) requires investigation. Both auditory [31] and haptic feedback via vibration [32] have been shown to influence the biomechanics of gait. 
The review demonstrates the success of real time feedback in improving propulsion biomechanics in both complete novices [18] and also experienced manual wheelchair users [13-16]. This indicates that real time feedback may be beneficial both in the early stages of wheelchair skills training and also to optimise an established technique. However, only one of the studies included in the review reported outcome at longer term follow up [14]. Therefore it is not possible to establish whether a single period of intervention is sufficient to influence technique in the long term. In addition, only one of the studies reports statistical power [15].

\section{Limitations}

The main limitation of the review is that due to the small number of articles included and the differences in terms of population recruited, type and form of intervention applied and outcomes measures recorded, a meta-analysis was not possible. In addition, the articles selected only consider the direct impact of real time feedback on temporal and kinetic push rim parameters. For further insight into minimising injury risk, the secondary impact of altering push rim variables on participant kinematics (joint angle and muscle activity levels) should be considered.

\section{Conclusion}

The findings of this review suggest that real time visual and haptic feedback can be used to modify wheelchair propulsion biomechanics. These results in conjunction with previous research investigating wheelchair propulsion and upper limb injury risk suggest that push arc and push rate may be the best parameters to target to optimise the fine balance between minimising peak force and task repetition. In addition, it appears that applying single variable feedback may be more successful than multiple variable feedbacks. However, these conclusions are drawn from data collected in the laboratory, 
mainly investigating the use of real time visual feedback. In reality, real time visual feedback is not a practical or safe option for the wheelchair user negotiating journeys outdoors. Further investigation is required to determine if the findings of the review can be applied during journeys outdoors and also if other forms of real time feedback, including auditory of haptic (vibration) can be successfully applied.

\section{Declaration of Interest Statement}

The authors report no conflicts of interest.

\section{References}

[1] Gutierrez DD, Thompson L, Kemp B, et al. The Relationship of Shoulder Pain Intensity to Quality of Life, Physical Activity, and Community Participation in Persons With Paraplegia. J. Spinal Cord Med. 2007;30:251-255.

[2] Preservation of Upper Limb Function Following Spinal Cord Injury. J. Spinal Cord Med. 2005;28:434-470.

[3] Boninger ML, Souza AL, Cooper RA, et al. Propulsion patterns and pushrim biomechanics in manual wheelchair propulsion. Arch. Phys. Med. Rehabil. 2002;83:718-723.

[4] Sawatzky B, DiGiovine C, Berner T, et al. The Need for Updated Clinical Practice Guidelines for Preservation of Upper Extremities in Manual Wheelchair Users: A Position Paper. Am. J. Phys. Med. Rehabil. 2015;94:313-324.

[5] MacPhee AH, Kirby RL, Coolen AL, et al. Wheelchair skills training program: a randomized clinical trial of wheelchair users undergoing initial rehabilitation1. Arch. Phys. Med. Rehabil. 2004;85:41-50.

[6] Öztürk A, Ucsular FD. Effectiveness of a wheelchair skills training programme for community-living users of manual wheelchairs in Turkey: a randomized controlled trial. Clin. Rehabil. 2011;25:416-424.

[7] Cowan RE, Boninger ML, Sawatzky BJ, et al. Preliminary Outcomes of the SmartWheel Users' Group Database: A Proposed Framework for Clinicians to Objectively Evaluate Manual Wheelchair Propulsion. Arch. Phys. Med. Rehabil. 2008;89:260-268. 
[8] Kr F, Ca D, Gd M, et al. Real-time biofeedback to target risk of anterior cruciate ligament injury: a technical report for injury prevention and rehabilitation. J. Sport Rehabil. [Internet]. 2014 [cited 2016 Nov 1];Technical Notes 13.

Available from: http://europepmc.org/abstract/med/24959871.

[9] Philip Crowell H, Milner CE, Hamill J, et al. Reducing Impact Loading During Running With the Use of Real-Time Visual Feedback. J. Orthop. Sports Phys. Ther. 2010;40:206-213.

[10] Moher D. Preferred Reporting Items for Systematic Reviews and MetaAnalyses: The PRISMA Statement. Ann. Intern. Med. 2009;151:264.

[11] Downs SH, Black N. The feasibility of creating a checklist for the assessment of the methodological quality both of randomised and non-randomised studies of health care interventions. J. Epidemiol. Community Health. 1998;52:377-384.

[12] Kloosterman MG, Snoek GJ, van der Woude LH, et al. A systematic review on the pros and cons of using a pushrim-activated power-assisted wheelchair. Clin. Rehabil. 2013;27:299-313.

[13] Blouin M, Lalumière M, Gagnon DH, et al. Characterization of the Immediate Effect of a Training Session on a Manual Wheelchair Simulator With Haptic Biofeedback: Towards More Effective Propulsion. IEEE Trans. Neural Syst. Rehabil. Eng. 2015;23:104-115.

[14] Rice IM, Pohlig RT, Gallagher JD, et al. Handrim Wheelchair Propulsion Training Effect on Overground Propulsion Using Biomechanical Real-Time Visual Feedback. Arch. Phys. Med. Rehabil. 2013;94:256-263.

[15] Richter WM, Kwarciak AM, Guo L, et al. Effects of Single-Variable Biofeedback on Wheelchair Handrim Biomechanics. Arch. Phys. Med. Rehabil. 2011;92:572-577.

[16] Degroot KK, Hollingsworth HH, Morgan KA, et al. The influence of verbal training and visual feedback on manual wheelchair propulsion. Disabil. Rehabil. Assist. Technol. 2009;4:86-94.

[17] Kotajarvi BR, Basford JR, An K-N, et al. The Effect of Visual Biofeedback on the Propulsion Effectiveness of Experienced Wheelchair Users. Arch. Phys. Med. Rehabil. 2006;87:510-515.

[18] de Groot S, Veeger HEJ, Hollander AP, et al. Consequence of feedback-based learning of an effective hand rim wheelchair force production on mechanical efficiency. Clin. Biomech. 2002;17:219-226. 
[19] Rankin JW, Kwarciak AM, Richter WM, et al. The influence of wheelchair propulsion technique on upper extremity muscle demand: A simulation study. Clin. Biomech. 2012;27:879-886.

[20] Boninger ML, Cooper RA, Baldwin MA, et al. Wheelchair pushrim kinetics: Body weight and median nerve function. Arch. Phys. Med. Rehabil. 1999;80:910-915.

[21] Boninger ML, Impink BG, Cooper RA, et al. Relation between median and ulnar nerve function and wrist kinematics during wheelchair propulsion1. Arch. Phys. Med. Rehabil. 2004;85:1141-1145.

[22] Boninger ML, Koontz AM, Sisto SA, et al. Pushrim biomechanics and injury prevention in spinal cord injury: Recommendations based on CULP-SCI investigations. J. Rehabil. Res. Dev. 2005;42:9-19.

[23] Boninger ML, Dicianno BE, Cooper RA, et al. Shoulder magnetic resonance imaging abnormalities, wheelchair propulsion, and gender. Arch. Phys. Med. Rehabil. 2003;84:1615-1620.

[24] Desroches G, Aissaoui R, Bourbonnais D. The Effect of Resultant Force at the Pushrim on Shoulder Kinetics During Manual Wheelchair Propulsion: A Simulation Study. IEEE Trans. Biomed. Eng. 2008;55:1423-1431.

[25] Bregman DJJ, Drongelen S van, Veeger HEJ. Is effective force application in handrim wheelchair propulsion also efficient? Clin. Biomech. 2009;24:13-19.

[26] Sonenblum SE, Sprigle S, Lopez RA. Manual Wheelchair Use: Bouts of Mobility in Everyday Life. Rehabil. Res. Pract. 2012;2012:e753165.

[27] Holloway CS, Symonds A, Suzuki T, et al. Linking wheelchair kinetics to glenohumeral joint demand during everyday accessibility activities. 201537 th Annu. Int. Conf. IEEE Eng. Med. Biol. Soc. EMBC. 2015. p. 2478-2481.

[28] Holloway C, Tyler N. A micro-level approach to measuring the accessibility of footways for wheelchair users using the Capability Model. Transp. Plan. Technol. 2013;36:636-649.

[29] Chow JW, Millikan TA, Carlton LG, et al. Kinematic and Electromyographic Analysis of Wheelchair Propulsion on Ramps of Different Slopes for Young Men With Paraplegia. Arch. Phys. Med. Rehabil. 2009;90:271-278.

[30] Morrow MMB, Kaufman KR, An K-N. Shoulder model validation and joint contact forces during wheelchair activities. J. Biomech. 2010;43:2487-2492. 
[31] Meardon SA, Derrick TR. Effect of step width manipulation on tibial stress during running. J. Biomech. 2014;47:2738-2744.

[32] Wheeler JW, Shull PB, Besier TF. Real-Time Knee Adduction Moment Feedback for Gait Retraining Through Visual and Tactile Displays. J. Biomech. Eng. 2011;133:41007. 
Records identified, Web of Science: 13, PubMed: 10, Science Direct: 151, Cochrane library: 2, IEEE Xplore: 105

Figure 1. Flow chart showing the literature search process. 
Table 1. Summary of eligible studies.

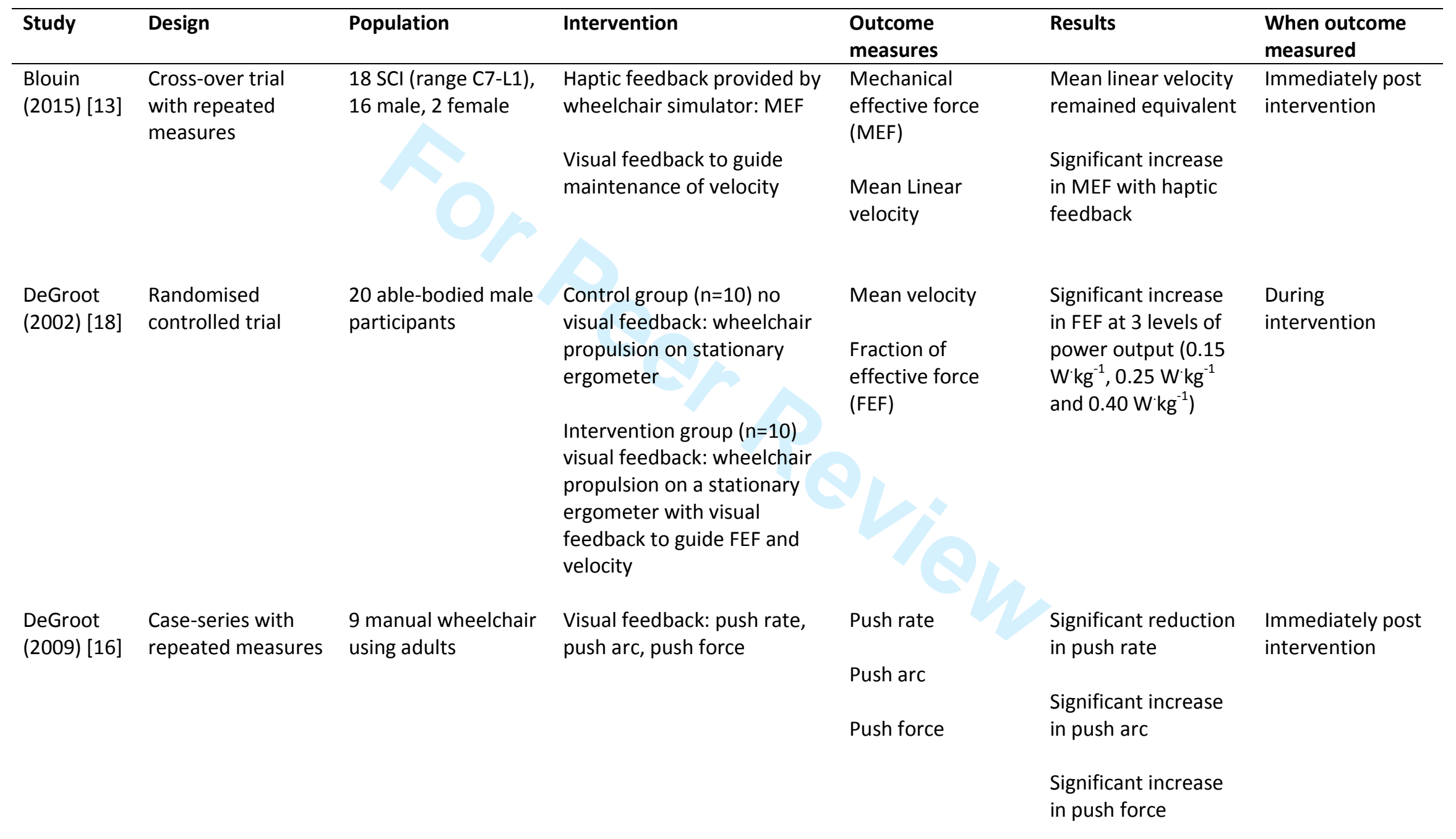




\begin{tabular}{|c|c|c|c|c|c|c|}
\hline $\begin{array}{l}\text { Kotajarvi } \\
\text { (2006) [17] }\end{array}$ & Controlled trial & $\begin{array}{l}18 \mathrm{SCl} \text { (range T4-L2), } \\
16 \text { male, } 2 \text { female }\end{array}$ & $\begin{array}{l}\text { Visual feedback: FEF, } \\
\text { propulsion velocity, power } \\
\text { output }\end{array}$ & $\begin{array}{l}\text { FEF } \\
\text { Velocity }\end{array}$ & $\begin{array}{l}\text { No significant } \\
\text { difference in FEF at } 2 \\
\text { levels of power } \\
\text { output }\end{array}$ & $\begin{array}{l}\text { During } \\
\text { intervention }\end{array}$ \\
\hline $\begin{array}{l}\text { Rice (2013) } \\
\text { [14] }\end{array}$ & $\begin{array}{l}\text { Randomised } \\
\text { controlled trial }\end{array}$ & $\begin{array}{l}27 \mathrm{SCl} \text { (range C7-L3), } \\
24 \text { male, } 3 \text { female }\end{array}$ & $\begin{array}{l}\text { Control group }(n=9) \text { : } \\
\text { Wheelchair propulsion on a } \\
\text { dynamometer } \\
\text { Instruction group ( } n=9 \text { ): } \\
\text { Multimedia presentation } \\
\text { then propel on } \\
\text { dynamometer } \\
\text { Real-time visual feedback } \\
\text { group ( } n=9) \text { : Multimedia } \\
\text { presentation then propel on } \\
\text { dynamometer with real-time } \\
\text { visual feedback: push rate, } \\
\text { push arc, propulsion velocity }\end{array}$ & $\begin{array}{l}\text { Push rate } \\
\text { Push arc } \\
\text { Propulsion velocity }\end{array}$ & $\begin{array}{l}\text { Push rate: significant } \\
\text { decrease vs. control } \\
\text { group at short and } \\
\text { long term follow up } \\
\text { and vs. instruction } \\
\text { group at long term } \\
\text { follow up } \\
\text { Push arc: significant } \\
\text { increase in push arc } \\
\text { vs. control group at } \\
\text { short and long term } \\
\text { follow up and vs. } \\
\text { instruction group at } \\
\text { long term follow up }\end{array}$ & $\begin{array}{l}\text { Immediately post } \\
\text { intervention and } \\
\text { at three months } \\
\text { follow up }\end{array}$ \\
\hline $\begin{array}{l}\text { Richter } \\
\text { (2011) [15] }\end{array}$ & $\begin{array}{l}\text { Case-series with } \\
\text { repeated measures }\end{array}$ & $\begin{array}{l}31 \text { manual } \\
\text { wheelchair users } \\
\text { (SCI, Spina Bifida, CP, } \\
\text { Spinal lipoma), } 27 \\
\text { male, } 4 \text { female }\end{array}$ & $\begin{array}{l}\text { Visual feedback: push rate, } \\
\text { push arc, peak force, braking } \\
\text { moment, push distance, } \\
\text { smoothness (separate trial } \\
\text { for each variable aiming for } \\
\text { maximum and } 10 \% \text { change) }\end{array}$ & $\begin{array}{l}\text { Push rate } \\
\text { Push arc } \\
\text { Peak force } \\
\text { Braking moment } \\
\text { Push distance } \\
\text { Smoothness }\end{array}$ & 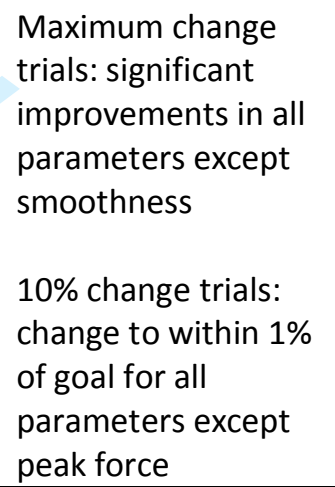 & $\begin{array}{l}\text { During } \\
\text { intervention }\end{array}$ \\
\hline
\end{tabular}


Table 2. Methodological quality according to modified Downs and Black checklist [11].

\begin{tabular}{lcccccc}
\hline Paper & Reporting & External validity & Internal validity & Power & Total \\
& & & Bias & Confounding & \\
& & & & & \\
\hline Maximum score & 11 & 3 & 7 & 6 & 1 & 28 \\
\hline Blouin (2015) [13] & 7 & 0 & 4 & 1 & 0 & 12 \\
DeGroot (2002) [18] & 9 & 0 & 6 & 4 & 0 & 19 \\
DeGroot (2009) [16] & 8 & 0 & 4 & 2 & 0 & 14 \\
Kotajarvi (2006) [17] & 8 & 1 & 6 & 1 & 0 & 16 \\
Rice (2013) [14] & 8 & 1 & 4 & 4 & 0 & 17 \\
Richter (2011) [15] & 7 & 0 & 4 & 1 & 1 & 13 \\
\hline
\end{tabular}

URL: http:/mc.manuscriptcentral.com/dandr Email: davemuller@suffolk.ac.uk 


\section{Implications for Rehabilitation}

- Upper limb pain and injuries are common secondary disorders that negatively affect wheelchair users' physical activity and quality of life

- Clinical guidelines suggest that manual wheelchair users should aim to propel with a semi-circular pattern with low a push rate and large push arc in the range in order to minimise upper limbs' loading

- Real time visual and haptic feedback are effective tools for improving propulsion biomechanics in both complete novices and experienced manual wheelchair users 